\title{
КВАНТОВО-ХИМИЧЕСКОЕ ИССЛЕДОВАНИЕ 2D АЛЛОТРОПОВ КАРБИДА КРЕМНИЯ
}

\author{
(C) 2017 А. В. Тучин, Л. А. Битюцкая, А. В. Калашников, Е. Н. Бормонтов \\ Воронежский государственньий университет, Университетская площадь, 1, 394018 Воронеж, Россия \\ e-mail: a.tuchin@bk.ru
}

Поступила в редакцию 14.08.2017

\begin{abstract}
Аннотация. Методами квантовой химии проведено исследование электронной структуры аллотропов 2D SiC с числом слоев $n=1-3$. Установлено, что 2D структуры карбида кремния образуют семейство полупроводниковых материалов с шириной запрещенной зоны от 1.132 до 2.150 эВ, а послойный рост структур определяет изменение типа проводника от прямозонного однослойного $\mathrm{SiC}$ к непрямозонному при числе слоев $n=2,3$. Исключением являются метастабильные структуры с шириной прямозонного перехода 1.339 и 1.132 эВ.
\end{abstract}

Ключевые слова: $\mathrm{SiC}, 2 \mathrm{D}$ аллотропы, квантово-химическое моделирование, электронная структура.

\section{ВВЕДЕНИЕ}

В последнее время наметилась тенденция перехода к некремниевой электронике. Так в качестве альтернативы кремнию в силовой и некоторых областях СВЧ электроники рассматривается карбид кремния. Интерес к этому полупроводниковому соединению определяется высокой механической прочностью (9.2-9.3 по шкале МООСА), широким диапазоном рабочих температур (теоретически, вплоть до $\left.1000{ }^{\circ} \mathrm{C}\right)$, высокой температурой Дебая $\left(\sim 1200{ }^{\circ} \mathrm{C}\right)$, наличием собственного окисла $\left(\mathrm{SiO}_{2}\right)$.

Карбид кремния в этих областях электроники постепенно вытесняет кремниевые устройства, обладая более привлекательными электрофизическими характеристиками $[2,5,6]$, а уникальные свойства данного соединения позволяют создавать сенсоры и датчики новых типов $[4,7,8]$. Особенный интерес для получения качественных гетероструктур представляют подложки $\mathrm{SiC}$, позволяющие получать бездефектные эпитаксиальные пленки GaN [11].

Несмотря на все достоинства, технологичность $\mathrm{SiC}$ электронного качества все еще далека от идеала. Высокая температура плавления-кристаллизации $\left(\sim 2200{ }^{\circ} \mathrm{C}\right)$ обуславливает малый размер бездефектной области кристалла (при температуре $\sim 2300{ }^{\circ} \mathrm{C}$ начинается диссоциация молекул $\mathrm{SiC}$ на исходные компоненты) и низкую скорость роста объемного кристалла, что, в свою очередь, сказыва- ется на дороговизне технологического оборудования и сопровождается огромными энергетическими и экономическими затратами при производстве.

Один из путей решения данной проблемы - переход к технологии микрокристаллического карбида кремния [22, 23, 24, 25], где не требуется прецизионный контроль температур, а сам процесс синтеза $\mathrm{SiC}$ значительно проще. Кроме того, наличие размерно-зависимых свойств у $\mathrm{SiC}$ (увеличение механической плотности, усиление интенсивности люминесценции [8], повышенная стойкость к радиационной эрозии) обуславливают все возрастающий интерес к изучению способов получения и параметров низкоразмерных структур на основе $\mathrm{SiC}$ [22-29]. После экспериментального получения графена и исследования его уникальных свойств особенно актуальным становится поиск графеноподобных 2D материалов. Многослойные структуры на основе $\mathrm{SiC}$ рассматриваются как одни из наиболее перспективных материалов наноэлектроники, т. к. в отличие от графена, они обладают не нулевой шириной запрещенной зоны, достигающей 3.72 эВ, и подходят для создания гетероструктур на их основе [7-12].

При переходе к 2D SiC актуальной становится задача исследования более широкого круга политипных модификаций и процессов фазовых превращений между ними [13-15]. Решение данной проблемы открывает путь к синтезу слоистых сверхрешеток, свойства которых определяются порядком и чередо- 
ванием слоев карбида кремния. Поэтому актуальной является задача исследования структурных особенностей и стабильности 2D аллотропов карбида кремния. Целью работы является установление закономерностей перестройки электронной структуры 2D аллотропов карбида кремния при последовательном изменении числа и конфигурации слоев.

\section{МЕТОДИКА РАСЧЕТА}

Объектами исследования являлись 2D структуры карбида кремния, с различной конфигурацией и числом слоев в интервале от 1 до 3 (рис. 1). Каждый слой представлял собой гексагональную графеноподобную сетку, составленную из последовательно чередующихся атомов углерода и кремния. Векторы трансляции $\mathbf{a}_{1}$ и $\mathbf{a}_{2}$ направлены вдоль двух смежных сторон гексагонов. Рассмотрены следующие аллотропные модификации 2D карбида кремния (представлено строение до проведения процесса оптимизации геометрии):

1. Структуры типа $A A A$, конфигурация атомов во всех слоях одинаковая.

2. В структурах типа $A \bar{A} A$, атомы углерода и кремния следующего слоя располагаются напротив атомов кремния и углерода второго слоя.

3. Структуры $A \bar{A}^{\prime} A$ получены из $A \bar{A} A$ смещением среднего слоя по одному из векторов трансляции на длину связи $\mathrm{R}_{\mathrm{Si-C}}$

4. Структуры $A A^{\prime} A$ получены из $A A A$ смещением среднего слоя по одному из векторов трансляции на длину связи $\mathrm{R}_{\mathrm{Si-C}}$

5. Структуры типа $A B A$ получены из $A A A$ смещением среднего слоя на вектор $\left[\frac{1}{2} R_{\mathrm{Si-C}} ; \frac{1}{2} R_{\mathrm{Si}-\mathrm{C}}\right]$

6. Структуры типа $A \bar{B} A$ получены из $A \bar{A} A$ смещением среднего слоя на вектор $\left[\frac{1}{2} R_{\mathrm{Si}-\mathrm{C}} ; \frac{1}{2} R_{\mathrm{Si-C}}\right]$.

Квантово-химическое моделирование электронной структуры 2D аллотропов карбида кремния проводилось теорией функционала электронной плотности (DFT) в приближении локальной спиновой плотности (LSDA) [11] с использованием валентно-расщепленного базиса $3-21 \mathrm{G}$ и числом $k$-точек 80000. Данный метод широко используется для моделирования многослойных 2D наноматериалов, в том числе мультиграфена $[12,13]$. Расчеты выполнены в Суперкомпьютерном центре Воронежского государственного университета с использованием специализированного программного комплекса для решения квантово-химических задач Gaussian09.
На первом этапе проводилась оптимизация геометрии каждой из 13 структур. Далее рассчитывались внутрислойные длины связей между атомами кремния и углерода $\left(R_{S i-C}\right)$, межслоевые расстояния $\left(R_{s h}\right)$, приведенная энергия связи $\left(E_{b}\right)$, межслоевая энергия связи $\left(E_{b s h}\right)$, ширина запрещенной зоны $\left(E_{g}\right)$.

Приведенная энергия связи рассчитывалась по формуле:

$$
E_{b}=\frac{E_{t o t}\left(\mathrm{Si}_{N} \mathrm{C}_{N}\right)-N E_{t o t}(\mathrm{Si})-N E_{t o t}(\mathrm{C})}{2 N},
$$

где $E_{t o t}(\mathrm{Si}), E_{t o t}(\mathrm{C}), E_{t o t}\left(\mathrm{Si}_{N} \mathrm{C}_{N}\right)$ - полная энергия уединенного атома кремния, углерода и структуры, содержащей $N$ атомов кремния и $N$ атомов углерода.

Приведенная межплоскостная энергия связи рассчитывалась по формуле:

$$
E_{b s h}=\frac{E_{t o t}\left(\mathrm{Si}_{N} \mathrm{C}_{N}\right)-n E_{t o t}(\mathrm{SiC})}{n},
$$

где $E_{t o t}(\mathrm{SiC}), E_{t o t}\left(\mathrm{Si}_{N} \mathrm{C}_{N}\right)-$ полная энергия однослойного карбида кремния и $n$-слойной структуры, содержащей $N$ атомов кремния и $N$ атомов углерода.

Оригинальность использованного в работе подхода к изучению электронной структуры многослойных аллотропов карбида кремния заключается в учете перестройки электронной структуры и геометрии при изменении числа и конфигурации слоев.

\section{РЕЗУЛЬТАТЫ И ОБСУЖДЕНИЕ}

Рассмотрим результаты моделирования однослойного $\mathrm{SiC}$. Длина химической связи $R_{\mathrm{Si-C}}$ составляет $1.80 \AA$, что на $0.38 \AA$ больше длины связи углерод-углерод в графене. Приведенная энергия связи $E_{b}=8.322$ эВ, что указывает на высокую термодинамическую стабильность данной структуры (табл. 1). При анализе электронного строения установлено, что однослойный $\mathrm{SiC}$ является прямозонным полупроводниковым материалом с шириной запрещенной зоны $E_{g} \approx 2$ эВ.

При последовательном увеличении числа слоев $n$ до 2 и 3 с конфигурацией $A A A$ параметры внутрислойных химических связей практически не изменяются, длина связи составляет $R_{\mathrm{Si-C}}=1.80$ $\AA$ А. Однако установлено смещение слоев на вектор $\left[\frac{1}{2} R_{\mathrm{Si}-\mathrm{C}} ; \frac{3}{4} R_{\mathrm{Si}-\mathrm{C}}\right]$ (рис. $1 g$ ), а также уменьшение межслоевых расстояний с $R_{s h}=3.38$ и $3.19 \AA$ для двух и трехслойного $\mathrm{SiC}$, что согласуется с ростом межслоевой энергии связи с $E_{b s h}=0.15$ и 0.22 эВ/слой. Таким образом, при послойном росте структуры 

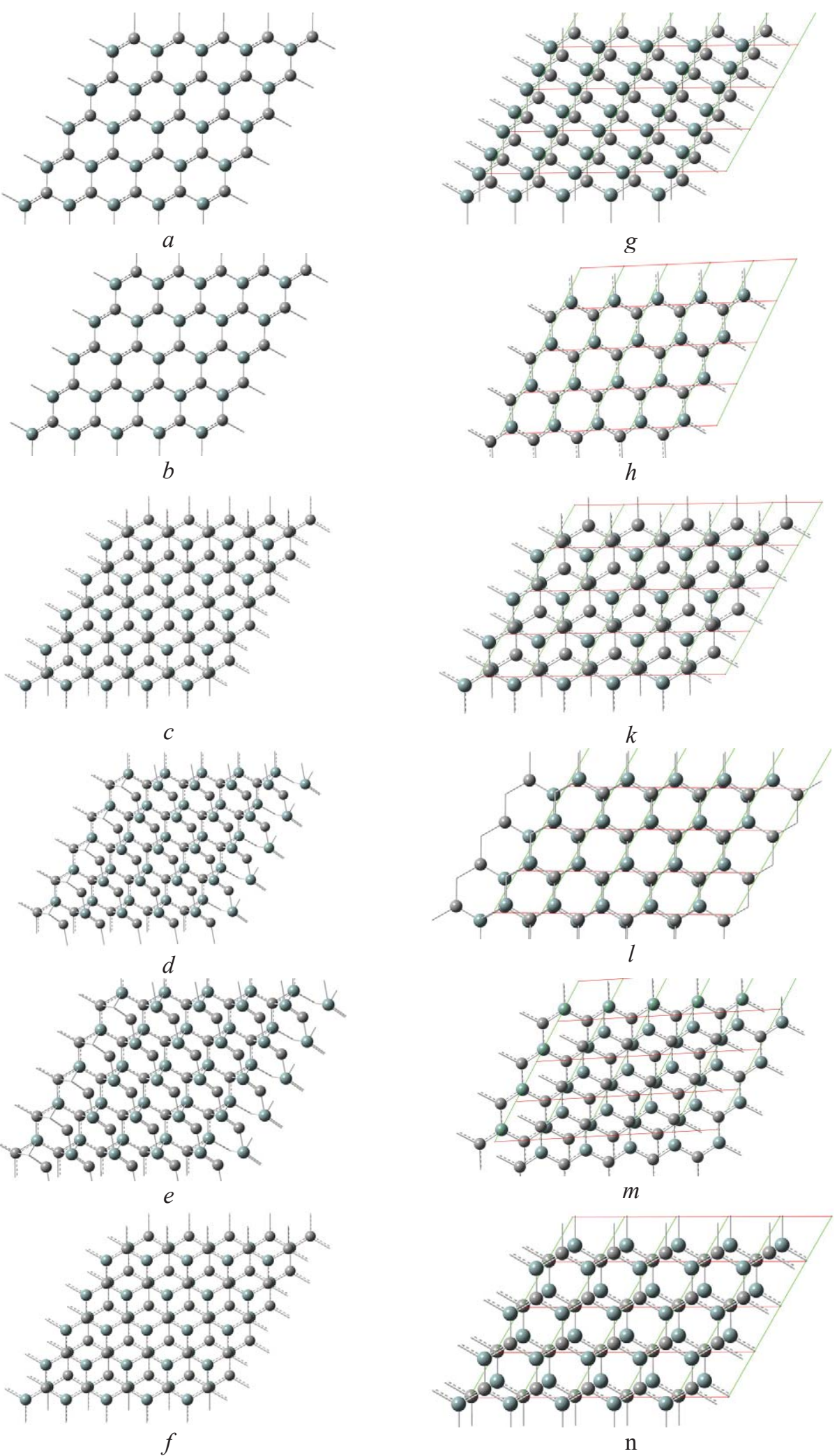

Рис. 1. Фрагменты 2D-аллотропов $\mathrm{SiC}: A A A(a), A \bar{A} A(b), A B A(c), A \bar{A}^{\prime} A(d), A A^{\prime} A(e), A \bar{B} A(f)$ до $(a-f)$ и после $(g-n)$ проведения процесса оптимизации геометрии

[Fig. 1. Fragments of 2D SiC allotropes: $A A A(a), A \bar{A} A(b), A B A(c), A \bar{A}^{\prime} A(d), A A^{\prime} A(e), A \bar{B} A(f)$ before $(\mathrm{a}-\mathrm{f})$ and after $(\mathrm{g}-\mathrm{n})$ geometry optimization] 
Таблица 1. Электрофизические свойства $2 \mathrm{D}$ карбида кремния в зависимости от числа и конфигурации слоев $\left(R_{\mathrm{Si}-\mathrm{C}}-\right.$ длина связи между атомами углерода и кремния в пределах слоя; $R_{s h}-$ межслоевое расстояние, $E_{b}-$ приведенная энергия связи; $E_{b s h}-$ приведенная межплоскостная энергия связи; $E_{g}^{d i r}, E_{g}^{\text {indir }},-$ ширина запрещенной зоны прямозонного и непрямозонного переходов)

[Table 1. Electrophysical properties of $2 \mathrm{D} \mathrm{SiC}$ in dependence of the layer number and configuration $\left(R_{\mathrm{Si}-\mathrm{C}}-\right.$ intralayer bond length carbon-silicium; $R_{s h}$ - interlayer distance, $E_{b}$ - reduced binding energy; $E_{b s h}-$ reduced interlayer binding energy; $E_{g}$ dir,$E_{g}^{\text {indir }}$ - direct and indirect energy gap)]

\begin{tabular}{|c|c|c|c|c|c|c|c|}
\hline $\begin{array}{c}\text { Исходная структура } \\
\text { [Initial structure] }\end{array}$ & $\begin{array}{c}\text { Кол-во } \\
\text { слоев } n \\
{[\text { Number of }} \\
\text { layers, } n]\end{array}$ & $R_{S i-C}, \AA$ & $R_{s h}, \AA$ & $\begin{array}{c}E_{b}, \\
\text { эB/aToм } \\
{[\mathrm{eV} / \text { atom }]}\end{array}$ & $\begin{array}{c}E_{b s h}, \\
\text { эВ/слой } \\
\text { [eV/layer] }\end{array}$ & $\begin{array}{c}E_{g}^{d i r} \\
\ni \mathrm{B} \\
{[\mathrm{eV}]}\end{array}$ & $\begin{array}{c}E_{g}^{\text {indir }}, \\
\text { ЭB } \\
{[\mathrm{eV}]}\end{array}$ \\
\hline$A$ & 1 & 1.805 & - & 8.322 & - & 1.998 & 1.998 \\
\hline$A A$ & 2 & 1.804 & 3.380 & 8.399 & 0.154 & 1.445 & 1.366 \\
\hline$A A A$ & 3 & 1.804 & 3.190 & 8.434 & 0.224 & 1.134 & 1.134 \\
\hline$A \bar{A}$ & 2 & 1.846 & 2.200 & 8.444 & 0.242 & 3.075 & 2.150 \\
\hline$A \bar{A} A$ & 3 & 1.837 & 2.334 & 8.524 & 0.403 & 2.175 & 1.568 \\
\hline$A B$ & 2 & 1.805 & 3.243 & 8.401 & 0.156 & 1.339 & 1.341 \\
\hline$A B A$ & 3 & 1.804 & 3.174 & 8.433 & 0.223 & 1.132 & 1.132 \\
\hline$A \bar{A}^{\prime}$ & 2 & 1.805 & 2.673 & 8.280 & -0.084 & 1.484 & 1.386 \\
\hline$A \bar{A}^{\prime} A$ & 3 & 1.804 & 3.156 & 8.316 & -0.014 & 1.344 & 1.325 \\
\hline$A A^{\prime}$ & 2 & 1.843 & 2.214 & 8.324 & 0.004 & 3.032 & 2.151 \\
\hline$A A^{\prime} A$ & 3 & 1.836 & 2.428 & 8.408 & 0.171 & 2.170 & 1.565 \\
\hline$A \bar{B}$ & 2 & 1.806 & 3.520 & 8.377 & 0.120 & 1.468 & 1.447 \\
\hline
\end{tabular}

типа $A-A A-A A A$ происходит ее стабилизация за счет увеличения межслоевой энергии связи. Рост структуры сопровождается уменьшением ширины запрещенной зоны и изменением типа полупроводника от прямозонного при $n=1$ к непрямозонному с $E_{g}=1.366$ и 1.133 эВ при $n=2$ и 3.

В результате оптимизации исходная симметрия упаковки $A \bar{A} A$ сохраняется (рис. $1 h$ ). Последовательный рост от одно- до трехслойной структуры определяет заметное перераспределение электронной плотности, определяющей увеличение внутрислойных длин связей $R_{\mathrm{Si}-\mathrm{C}}=1.80 \AA(n=1)$ до $R_{\mathrm{Si}-\mathrm{C}}=1.85$ и $1.84 \AA(n=2$ и $n=3)$, а также чувствительность эффективного заряда атомов к числу слоев: для структуры с $n=2$ эффективные заряды атомов кремния и углерода равны по модулю, для трехслойной структуры установлено увеличение модуля эффективного заряда внешних слоев относительно среднего. Несмотря на удлинение химических связей, приведенная энергия связи в упаковке $A \bar{A}$ и $A \bar{A} A$ ( $E_{b}=8.443,8.524$ эВ/атом) больше, чем в $A A$ и $A A A\left(E_{b}=8.322,8.399\right.$ эВ/атом $)$ (табл. 1). Стабилизация структуры происходит за счет почти двукратного увеличения межслоевой энергии связи $E_{b s h}=242$ и 403 мэВ для структур
$A \bar{A}$ и $A \bar{A} A$, против $E_{b s h}=154$ и 224 мэВ для $A A$ и $A A A$. Образование дополнительных межслоевых связей между атомами кремния и углерода определяет уменьшение расстояния между слоями на 1.18 и $0.86 \AA$ (при числе слоев $n=2,3)$, а также увеличение ширины запрещенной зоны до рекордных значений среди всех рассмотренных аллотропных модификаций: $E_{g}=2.150$ и 1.568 эВ для структур $A \bar{A}$ и $A \bar{A} A$. Обе структуры являются непрямозонными полупроводниками.

Несмотря на то, что структуры типа $A B A$ с конфигурацией слоев соответствующей графиту значительно отличаются по строению от структур $A A A$, их параметры достаточно близки: внутрислойные длины связей $R_{S i-C} \sim 1.8 \AA$, межслоевые расстояния $R_{s h}$ на 0.014 и $0.001 \AA$ короче, что определяется незначительным отличием межслоевых энергий связей (табл. 1). Структуры характеризуется неравномерным распределением удельного заряда, ввиду топологической не инвариантности расположения атомов. В отличие от двух типов упаковок, рассмотренных ранее, структуры типа $A B A$ являются прямозонными полупроводниками. Ширина запрещенной зоны составляет $E_{g}=1.339$ и 1.132 эВ. 
Поскольку среди всех исследованных структур наиболее стабильными являются $A \bar{A}$ и $A \bar{A} A$, то среди метастабильных структур проявляется конкуренция между укладкой слоев по типу $A A A$ и $A B A$. Поскольку второй тип является прямозонным полупроводником и наиболее интересен для разработки устройств наноэлектроники, то в технологии 2D SiC структур необходимо учесть, что для двухслойного $\mathrm{SiC} A B$ упаковка является ближайшим возбужденным состоянием относительно основного $A \bar{A}$, а в трехслойной структуре $A B A$ является вторым возбужденным состоянием после $A \bar{A} A$ и $A A A$.

Еще менее стабильными являются структуры типа $A \bar{A}^{\prime}, A \bar{A}^{\prime} A, A A^{\prime} A A^{\prime} A$. Приведенные энергии связи составляют $8.280,8.316$ и $8.324,8.408$ эВ/атом соответственно. Отметим, что послойный рост первой структуры сопровождается ее дестабилизацией относительно однослойного $\mathrm{SiC}$. Чтобы сохранить упаковку структуры, необходима энергия 84 и 14 мэВ/атом. Послойный рост второй структуры от $n=1$ до 3 характеризуется изменением параметров химической связи: $R_{\mathrm{Si}-\mathrm{C}}=1.805,1.843$, $1.836 \AA, E_{b}=8.322,8.322,8.408$ эВ/атом. Ширина непрямозонного перехода составляет $E_{g}=1.998$, 2.151, 1.565 эВ.

Для структуры $A \bar{B}$ установлено смещение одного слоя на вектор $\left[\frac{2}{3} R_{\mathrm{Si}-\mathrm{C}} ; \frac{2}{3} R_{\mathrm{Si}-\mathrm{C}}\right]$ относительно исходной геометрии. Данная структура характеризуется максимальным в сравнении с другими межслойным расстоянием $R_{S H} \sim 3.5 \AA$. Данный политип $2 \mathrm{D} \mathrm{SiC} \mathrm{является} \mathrm{прямозонным} \mathrm{полупроводником} \mathrm{с}$ шириной запрещенной зоны $E_{g}=1.44$ эВ.

Отдельно остановимся на вопросе стабильности аллотропов 2D карбида кремния. Наиболее стабильными являются структуры с упаковкой $A \bar{A} A$, что определяется формированием дополнительных ковалентных полярных связей между слоями. Поэтому данные структуры характеризуются минимальными межплоскостными расстояниями и максимальной шириной запрещенной зоны. Остальные пять структурных типов $A A A, A \bar{A}^{\prime} A, A A^{\prime} A, A B A$, $A \bar{B} A$ являются метастабильными и характеризуются конкуренцией между ними при послойном росте из-за близости значений полных энергий. Это определяет технологические трудности получения карбида кремния определенного политипа. Между тем, именно структуры $A B A$ с числом слоев $n=2,3$ являются прямозонными полупроводниками с шириной запрещенной зоны 1.339 и 1.132 эВ соответственно. Данные метастабильные структуры стабильнее структур с упаковкой $A \bar{A}^{\prime} A, A A^{\prime} A, A \bar{B} A$, что опре- деляет актуальность поиска технологических способов стабилизации данного политипа. Поскольку геометрия расположения атомов соответствует графиту и мультиграфену, перспективным направлением является исследование свойств и технологий гетероструктур графен/2D SiC, как материалов с возможной стабилизацией фазы $A B A \mathrm{SiC}$.

\section{ЗАКЛЮЧЕНИЕ И ВЫВОДЫ}

В ходе выполнения работы проведено численное моделирование электронной структуры аллотропов 2D SiC с числом слоев от 1 до 3. Установлено, что многослойные структуры карбида кремния образуют семейство полупроводниковых материалов с шириной запрещенной зоны от 1.132 до 2.150 эВ, свойства которых определяются числом и конфигурацией слоев. Выявлено, что двух- и трехслойный $2 \mathrm{D}$ карбид кремния с упаковкой типа $A \bar{A} A$ является наиболее стабильным и обладает максимальной шириной запрещенной зоны 2.150 и 1.568 эВ среди исследованных аллотропных модификаций. Обнаружено, что послойный рост структур определяет изменение типа проводника от прямозонного однослойного $\mathrm{SiC}$ к непрямозонному при числе слоев $n=2,3$. Исключением являются метастабильные структуры типа $A B A$ с шириной прямозонного перехода 1.339 и 1.132 эВ. Схожее строение данной аллотропной модификации со строением мультиграфена является предпосылкой ее стабилизации

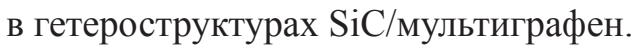

Работа выполнена при финансовой поддержке РФФИ (проект № 16-43-360281 p_a)

\section{СПИСОК ЛИТЕРАТУРЫ}

1. Полищук А. // Компоненты и технологии, 2004, № 5 , c. 20-23.

2. Рябинина И. А., Рембеза С. И., Рембеза Е. С. // Вестник ВГТУ, 2009, т. 5, № 12, с. 198-202.

3. Чепурнов В. И., Фидман Т. П. // Микросистемная техника, 2002, № 2, с. 17-21.

4. Гурин А. С., Печерская Р. М // Известия выстих учебных заведений. Поволжский регион. Технические науки, 2014, № 1(29), с. 46-53.

5. Лучинин В., Таиров Ю. // Современная электроника, 2009, № 7, с. 12-15.

6. Gundiah G., Madhav G. V., Govindaraj A., Seikh M., Rao C. N. R. // J. Mater. Chem., 2002, № 5, pp. 16061611.

7. Zhang Y., Wang L., He R., Chen X., Zhu J. // Solid State Communications, 2001, vol. 118, № 11, pp. 595-598.

8. Pan Z., Lai H. L., Au F. C. K., Duan X., Zhou W., Shi W., Wang N., Lee C. S., Wong N. B., Lee S. T., Xie S. // Adv. Mater., 2000, № 12, pp. 1186-1190. 
9. Александров П. А., Белова Н. Е., Демаков К. Д., Иванова Л. М., Кузнецов Ю. Ю., Степанов Н. В., Шемардов С. Г. // Вопросы атомной науки и техники. Сер. Термоядерный синтез, 2007, вып. 1, с. 68-75.

10. Емельченко Г. А., Масалов В. М., Жохов А. А., Максимук М. Ю., Фурсова Т. Н., Баженов А. В., Зверькова И. И., Хасанов С. С., Штейнман Э. А., Терещенко А. Н. // ФTT, 2011, т. 53, вып. 6, с. 1059-1063.

11. Каргин Ю. Ф., Ивичева С. Н., Лысенко А. С., Аладьев Н. А., Куцев С. В., Шворнева Л. И. // Неорганические материалы, 2009, т. 45, № 7, с. 820-828.

12. Борунов А. Б., Стрелецкий А. Н. Мудрецова С. Н., Леонов А. В., Бутягин П. Ю. // Коллоидный журнал, 2011, т. 73, № 5, с. 599-607.

13. Болотникова О. А. «Высокие технологии в современной науке и технике-2016», Сборник трудов V Международной научно-технической конференции молодых ученых, аспирантов и студентов, 5-7 декабря, Томск, 2016, с. 50-51.

14. Widmann M., Lee S.-Y., Rendler T., Son N. T., Fedder H., Paik S., Yang L.-P., Zhao N., Yang S., Booker I., Denisenko A., Momenzadeh S .A., Gerhardt I., Ohshima T., Gali A., Janzen E., Wrachtrup J. // Nature Material, 2015, № 14, pp. 164-168.
15. Morello A. // Nature Material, 2015, № 14, pp. 135136.

16. Kou L., Ma Y., Tan X., Frauenheim T., Du A., Smith S. // J. Phys. Chem. C, 2015, vol. 119, № 12, pp. 6918-6922.

17. Li P., Zhou R., Zeng X. C. // Nanoscale, 2014, vol. 6, pp. 11685-11691.

18. Kraus H., Soltamov V. A., Fuchs F., Simin D., Sperlich A., Baranov P. G., Astakhov G. V., Dyakonov V. // Scientific Reports, 2014, vol. 4, p. 5303(8).

19. Zhou H., Lin Z., Guo H., Lin S., Sun Y., Xu Y. // Journal of Semiconductors, 2017, vol. 38, № 3, 033002 (4).

20. Беленков Е. А., Агалямова Э. Н., Гришняков В. А. // ФTT, 2012, т. 54, № 2, с. 404-410.

21. Беленков Е. А., Агалямова Э. Н. // Вестник Челябинского государственного университета, Физика, 2009, т. 24, вып. 5, с. 13-21.

22. Агалямова Э. Н., Беленков Е. А., Гришнякова В.А. // Вестник Челябинского государственного университета, Физика, 2011, т. 15, вып. 10, с. 15-24.

23. Kasper Y., Tuchin A., Bokova A., Bityutskaya L. // J. of Phys., Conf. Series, 2016, vol. 741, № 1, p. 012022(5).

24. Бокова А. М., Тучин А. В., Битюцкая Л. А. // Известия вузов. Электроника, 2015, т. 20, № 1, с. 5-9.

\title{
QUANTUM-CHEMICAL STUDY OF 2D ALLOTROPES OF SILICON CARBIDE
}

\author{
(C) 2017 A. V. Tuchin, L. A. Bityutskaya, A. V. Kalashnikov, E. N. Bormontov \\ Voronezh State University, 1 Universitetskaya sq., 394018 Voronezh, Russia \\ e-mail:a.tuchin@bk.ru
}

Received 14.08.2017

\begin{abstract}
Multilayer structures based on $\mathrm{SiC}$ are considered to be among the most promising materials of nanoelectronics, since they have a non-zero bandgap width up to $3.72 \mathrm{eV}$ and can be used to produce heterostructures. The aim of the paper is to establish the dependency between the rearrangement of the electronic structure of 2D allotropes of silicon carbide and a successive change in the number and configuration of layers. The material of the study were single-layer silicon carbide and 6 allotropic modifications of $\mathrm{SiC}$ with the number of layers $n=2,3$. Quantum-chemical modelling of the electronic structure of 2D allotropes of silicon carbide was performed using the density functional theory (DFT) in the local spin density approximation (LSDA). It was established that multilayer structures of silicon carbide form a family of semiconductor materials with a bandgap width from 1.132 to $2.150 \mathrm{eV}$, whose properties are determined by the number and configuration of layers. It was also established that two- and three-layer 2D silicon carbide with thepackaging type $A \bar{A} A$ is the most stable among the analysed allotropic modifications and has a maximum band gap of 2.150 and $1.568 \mathrm{eV}$. It was found that layer-by-layer growth of structures determines a change in the type of the semiconductor from a direct bandgap single-layer $\mathrm{SiC}$ to an indirect bandgap with the number of layers $n=2,3$. An exception is the metastable $A B A$ structure with a direct bandgap of 1.339 and $1.132 \mathrm{eV}(n=2,3)$. Since this allotropic modification has a structure similar to the structure of multigraphene, it can be stabilized in $\mathrm{SiC} /$ multigrapheneheterostructures.
\end{abstract}

Keywords: SiC, 2D allotropes, quantum-chemical simulation, electronic structure. 


\section{ACKNOWLEDGEMENTS}

The reported study was supported by the Russian Foundation for Basic Research (project No. 16-43$360281 r_{-} a$ ).

\section{REFERENCES}

1. Polishhuk A. Components and Technologies, 2004, no. 5, pp. 20-23. (in Russian)

2. Rjabinina I. A., Rembeza S. I., Rembeza E. S. The Bulletin of Voronezh State Technical University, 2009, vol. 5, no. 12, pp. 198-202. (in Russian)

3. Chepurnov V. I., Fidman T. P. Microsystems Technology, 2002, no. 2, pp. 17-21. (in Russian)

4. Gurin A. S., Pecherskaja R. M. University Proceedings. Volga Region. Technical Sciences, 2014, no. 1 (29), pp. 46-53. Available at: http://izvuz_tn_eng.pnzgu.ru/files/ izvuz_tn_eng.pnzgu.ru/05.pdf (in Russian)

5. Luchinin V., Tairov Ju. Modern Electronics, 2009, no. 7, pp. 12-15. Available at: https://www.soel.ru/upload/ magazines/OPEN/SoEl_2009-7/SoEl_2009-7pr.html\#12 (in Russian)

6. Gundiah G., Madhav G. V., Govindaraj A., Seikh M., Rao C. N. R. J. Mater. Chem., 2002, no. 5, pp. 1606-1611. DOI: 10.1039/B20161F. Available at: http://pubs.rsc.org/en/ content/articlelanding/2002/jm/b200161f

7. Zhang Y., Wang L., He R., Chen X., Zhu J. Solid State Communications, 2001, vol. 118, no. 11, pp. 595-598. DOI: 10.1016/S0038-1098(01)00181-8. Available at: http://www. sciencedirect.com/science/article/pii/S0038109801001818 ?via\%3Dihub

8. Pan Z., Lai H. L., Au F. C. K., Duan X., Zhou W., Shi W., Wang N., Lee C. S., Wong N. B., Lee S. T., Xie S. Adv. Mater., 2000, 12, pp. 1186-1190. DOI: 10.1002/15214095(200008)12:16<1186::AID-ADMA1186>3.0.CO;2-F. Available at: http://onlinelibrary.wiley.com/ doi/10.1002/1521-4095(200008)12:16\%3C1186::AIDADMA1186\%3E3.0.CO;2-F/full

9. Aleksandrov P. A., Belova N. E., Demakov K. D., Ivanova L. M., Kuznecov Ju. Ju., Stepanov N. V., Shemardov S. G. Questions of Atomic Science and Technology. Ser. Thermonuclear Synthesis, 2007, 1, pp. 68-75. (in Russian)

10. Emel'chenko G. A., Masalov V. M., Zhohov A. A., Maksimuk M. Ju., Fursova T. N., Bazhenov A. V., Zver'kova I. I., Hasanov S. S., Shtejnman Je. A., Tereshhenko A. N. Physics of the Solid State, 2011, vol. 53, 6, pp.1121-1063 DOI: https://doi.org/10.1134/S1063783411060096. Available at: https://link.springer.com/article/10.1134/ S1063783411060096.

11. Kargin Ju. F., Ivicheva S. N., Lysenko A. S., Alad'ev N. A., Kucev S. V., Shvorneva L.I. Inorganic Materials, 2009, vol. 45, no. 7, p. 758. DOI: https://doi. org/10.1134/S0020168509070103. Available at: https://ink. springer.com/article/10.1134/S0020168509070103
12. Borunova A. B., Streleckii A. N. Mudretsova S. N., Leonov A. V., Butyagin P. Ju. Colloid Journal, 2011, vol. 73, no. 5, pp. 605-613. DOI: 10.1134/S1061933X 1104003X Available at: http://pleiades.online/cgi-perl/ search.pl?type $=$ abstract $\&$ name $=$ colljour\&number $=5 \&$ yea $\mathrm{r}=11$ \&page $=605$

13. Bolotnikova O. A. "High Technologies in Modern Science and Technology-2016". Proceedings of the V International Conference, December 5-7, Tomsk, 2016, pp. 5051. (in Russian)

14. Widmann M., Lee S.-Y., Rendler T., Son N. T., Fedder H., Paik S., Yang L.-P., Zhao N., Yang S., Booker I., Denisenko A., Momenzadeh S. A., Gerhardt I., Ohshima T., Gali A., Janzen E., Wrachtrup J. Nature Material, 2015, no. 14 , pp. $164-168$. DOI: $10.1038 /$ ncomms8783. Available at: https://www.nature.com/articles/ncomms8783

15. Morello A. Nature Material, 2015, no. 14, pp. 135136. DOI: $10.1038 / \mathrm{nmat} 4171$. Available at: http://www. nature.com/nmat/journal/v14/n2/full/nmat4171.html

16. Kou L., Ma Y., Tan X., Frauenheim T., Du A., Smith S. J. Phys. Chem. C, 2015, 119 (12), pp. 6918-6922. DOI: $10.1021 /$ acs.jpcc.5b02096 Available at: http://pubs. acs.org/doi/ipdf/10.1021/acs.jpcc.5b02096

17. Li P., Zhou R., Zeng X. C. Nanoscale, 2014, no. 6, pp. 11685-11691. DOI: 10.1039/C4NR03247K Available at: http://pubs.rsc.org/en/content/articlehtml/2014/nr/ c4nr03247k.

18. Kraus H., Soltamov V. A., Fuchs F., Simin D., Sperlich A., Baranov P. G., Astakhov G. V., Dyakonov V. Scientific Reports, 2014, vol. 4, 5303(8). DOI: 10.1038/ srep05303 Available at: https://www.nature.com/articles/ srep05303\#supplementary-information

19. Zhou H., Lin Z., Guo H., Lin S., Sun Y., Xu Y. Journal of Semiconductors, 2017, vol. 38, no. 3, 033002. DOI: 10.1088/1674-4926/38/3/033002 Available at: http://iopscience.iop.org/article/10.1088/1674-4926/38/3/033002

20. Belenkov E. A., Agaljamova Je. N., Grishnjakov V. A. Physics of the Solid State 2012, vol. 54, no. 2, pp. 433. DOI https://doi.org/10.1134/S1063783412020072 Available at: https://link.springer.com/article/10.1134/ S1063783412020072

21. Belenkov E. A., Agaljamova Je. N. Bulletin of the Chelyabinsk State University, Physics, 2009, no. 24 (162), 5, pp.13-21. (in Russian)

22. Agalyamova E. N., Belenkov E. A., Grishnyakova V. A. Bulletin of the Chelyabinsk State University, Physics, 2011, no. 15 (230), 10, pp. 15-24. (in Russian)

23. Kasper Y., Tuchin A., Bokova A., Bityutskaya L. J. of Phys., Conf. Series, 2016, vol. 741, no. 1, 012022(5). DOI: 10.1088/1742-6596/741/1/012022 Available at: http:// iopscience.iop.org/article/10.1088/1742-6596/741/ 1/012022/meta

24. Bokova A. M., Tuchin A. V., Bitjuckaja L. A. Proceedings of High Schools. Electronics, 2015, 20, no. 1, pp. 5-9. 
Тучин Андрей Витальевич - к. ф.-м. н., доцент кафедры физики полупроводников и микроэлектроники, Воронежский государственный университет; тел.: +7 (908) 1485775, e-mail: a.tuchin@bk.ru

Битюикая Лариса Александровна - к. х. н., доцент кафедры физики полупроводников и микроэлектроники, Воронежский государственный университет; тел.: +7 (473) 2208481, e-mail: me144@phys.vsu.ru

Калашников Андрей Васильевич - аспирант кафедры физики полупроводников и микроэлектроники, Воронежский государственный университет; тел.: +7(951) 5503243, e-mail: akalash49@gmail.com

Бормонтов Евгений Николаевич - д. ф.-м. н., профессор, заведующий кафедрой физики полупроводников и микроэлектроники, Воронежский государственный университет; тел.: +7 (473) 2208481, e-mail: me144@ phys.vsu.ru
Tuchin Andrei V. - Cand. Sci. (Phys.-Math.), Associate Professor of the Department of Physics of Semiconductors and Microelectronics, Voronezh State University; ph.: +7 (908) 1485775, e-mail: a.tuchin@bk.ru

Bityutskaya Larisa A. - Cand. Sci. (Chem.), Associate Professor of the Department of Physics of Semiconductors and Microelectronics, Voronezh State University; ph.: +7 (473) 2208481, e-mail: me144@phys.vsu.ru

Kalashnikov Andrei V. - graduate student, Department of Physics of Semiconductors and Microelectronics, Voronezh State University; ph.: +7(951) 5503243, e-mail: akalash49@gmail.com

Bormontov Eugene N. - Dr. Sci. (Phys.-Math.), Full Professor, Head of Department of Physics of Semiconductors and Microelectronics, Voronezh State University; ph.: +7 (473) 2208481, e-mail: me144@phys.vsu.ru 